\title{
REVIEW
}

\section{Exocytic Process Analyzed with Two-photon Excitation Imaging in Endocrine Pancreas}

\author{
NORIKO TAKAHASHI AND HARUO KASAI
}

Division of Biophysics, Center for Disease Biology and Integrative Medicine, Graduate School of Medicine, the University of Tokyo, 7-3-1 Hongo, Bunkyo-ku, Tokyo 113-0033, Japan

\begin{abstract}
To elucidate the spatiotemporal profiles of final secretory stage, we have established two-photon extracellular polar tracer (TEP) imaging, with which we can quantify all exocytic events in the plane of focus within the intact tissues. With such technique, we can estimate the precise diameters of vesicles independently of the spatial resolution of optical microscope, and measure the fusion pore dynamics at nanometer resolution. At insulin exocytosis in the pancreatic islets, it took two seconds for the fusion pore to dilate from $1.4 \mathrm{~nm}$ in diameter to $6 \mathrm{~nm}$ in diameter, and such unusual stability of the pore may be due to the crystallization of the intragranular contents. Opening of the pore was preceded by unrestricted lateral diffusion of lipids along the inner wall of the pores, supporting the idea that this structure was mainly composed of membrane lipids. TEP imaging has been also applied to other representative secretory glands, and has revealed hitherto unexpected diversity in spatial organizations of exocytosis and endocytosis, which are relevant for physiology and pathology of secretory tissues. In the pancreatic islet, compound exocytosis was characteristically inhibited $(<5 \%)$, partly due to the rarity of SNAP25 redistribution into the exocytosed vesicle membrane. Such mechanisms necessitate transport of insulin granules to the cell surface for fusion, and possibly rendering exocytosis more sensitive to metabolic state. Twophoton imaging will be powerful tools to elucidate molecular and cellular mechanisms of exocytosis and related disease, and to develop new therapeutic agencies as well as diagnostic tools.
\end{abstract}

Key words: Imaging, Secretion, Hormone, Diabetes mellitus, Insulin

(Endocrine Journal 54: 337-346, 2007)

PEPTIDE hormone is stored in secretory granules. Granules are transported toward target region of the plasma membrane. Then the granular contents are released to the extracellular space, via fusion of plasma membrane and vesicle membrane. Such final step of secretion is termed as exocytosis, and is one of the fundamental cellular mechanism $[1,2]$. Exocytosis is regulated by cytosolic $\mathrm{Ca}^{2+}$, cAMP and GTPase in endocrine cells, exocrine cells, hematopoietic cells and neurons [3, 4].

Key molecules involved in exocytosis have been identified in the last 15 years [5]. The fundamental mechanisms of these complex processes, however, are

Correspondence to: Noriko TAKAHASHI, Division of Biophysics, Center for Disease Biology and Integrative Medicine, Graduate School of Medicine, the University of Tokyo, 7-3-1 Hongo, Bunkyo-ku, Tokyo 113-0033, Japan still largely unknown. Direct measurement of individual steps of exocytosis itself is still a matter of challenge (Fig. 1). An ideal methodology should be able 1) to track sizes and histories of vesicles, 2) to reveal dynamics of hemifusion of two biological membranes, 3 ) to estimate the dynamics of the fusion pore that connect extracellular space and vesicles, 4) to detect the fates of $\Omega$-shaped profile of fused vesicles, and 5 ) to follow up compound exocytosis and endocytosis. Measurement of exocytosis in native and regenerated tissues is key to the development of new drugs and therapy. In this review, we introduce how two-photon excitation imaging of exocytosis is powerful to achieve these goals [6-14], and explain the importance of developing user-friendly two-photon microscope systems. 
a. Membrane capacitance measurement

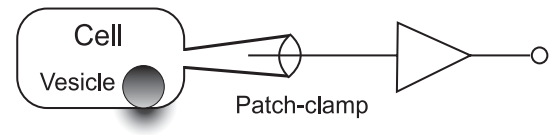

b. Electrochemical detection

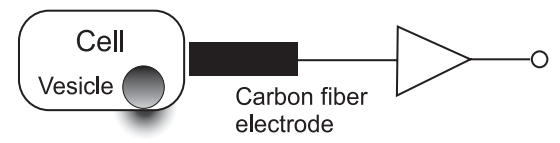

c. Imaging with prefusion labeling

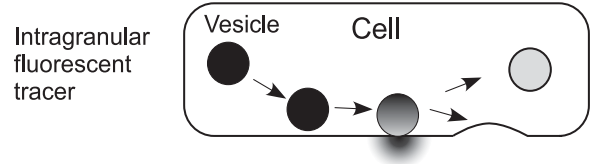

d. Imaging with postfusion labeling (TEP)

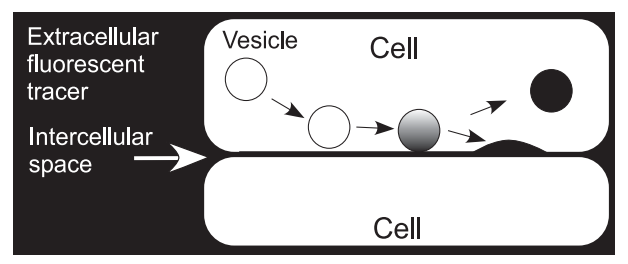

Fig. 1. Analytical methods to study exocytosis and endocytosis.

\section{Two-photon imaging with extracellular polar fluorescent tracer (TEP imaging)}

We have systematically developed a postfusion labeling method which can be generally applicable to any secretory preparation using a two-photon microscope (Fig. 1d). We gave this method a name, "Twophoton Extracellular-Polar-tracer (TEP) imaging”. To visualize insulin exocytosis, for example, we immersed the pancreatic islet preparations with a solution containing polar fluorescent tracers, sulforhodamine-B (SRB). The tracer was excited by laser-scanning microscopy with a mode-locked femtosecond-pulse Ti:sapphire laser (wavelength, $830 \mathrm{~nm}$ ), and we found mesh structures inside the islets (Fig. 2a) [15]. The three-dimensional reconstructed images suggested that the fluorescence reflected microvasculatures inside the islets. When we stimulated the islets with secretagogue such as $10-20 \mathrm{mM}$ glucose, exocytic event of individual insulin granule could be identified as the appearance of small fluorescent spot (mean diameter: $\sim 0.4 \mu \mathrm{m}$ full length at half maximum), reflecting the entry of extracellular polar fluorescent tracer a

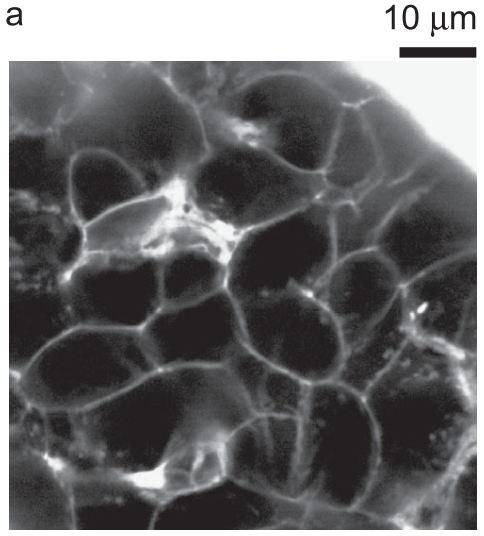

b

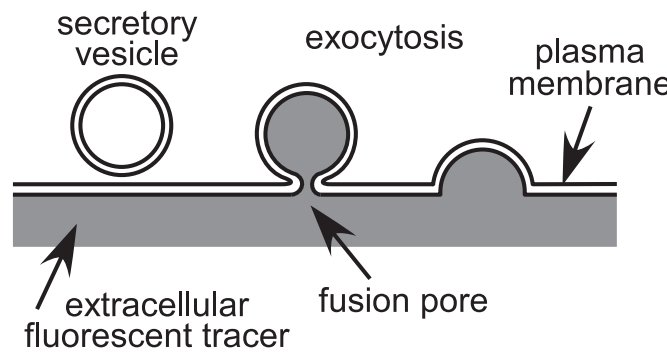

C

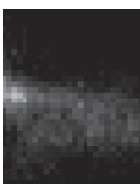

time (s)

0

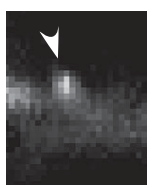

0.8

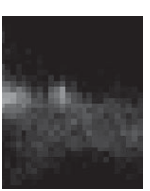

2.4

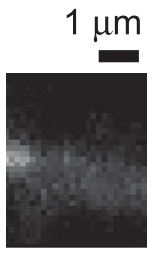

16.8

Fig. 2. TEP imaging of insulin exocytic events in intact pancreatic islets. a) Mouse pancreatic islet was immersed in a solution containing polar fluorescent tracer, SRB. TEP imaging revealed mesh structure, which was tubular revealed by three-dimensional reconstruction. b, c) Upon $20 \mathrm{mM}$ glucose stimulation, an exocytic event of single insulin vesicle was detected as the transient appearance of small fluorescent spots (arrowhead in c). 
into the fused vesicle (Fig. 2b, c). Most of the fluorescent spots disappeared within 10 seconds, reflecting full fusion of the fused vesicle membrane into the plasma membrane (Fig. 2c). We interpreted that the transient appearance of fluorescent spots reflected most of the individual exocytic events of insulin vesicles in the focal plane by the reasons described below: 1) the diameter of the spots were similar with diameter of insulin vesicles, 2) after chemical fixation, the fluorescent spots overlapped with the puncta those were positive in insulin immunohistochemistry, 3) fluorescent spots started to appear just when the cytosolic calcium concentration increased upon high glucose administration, 4) forskolin, which is well known to enhance insulin secretion, increased the frequency of appearance of the spots, and 5) the frequency of the appearance of spots could explain the amount of insulin secretion measured by RIA $[7,13]$.

\section{Advantages of TEP imaging}

TEP imaging can be readily performed by commercially available two-photon microscopes. The highly quantitative nature of TEP imaging takes advantage of a unique combination of the following three factors: 1) two-photon excitation imaging, 2) the usage of extracellular polar tracers and 3) the narrow intercellular spaces of intact tissues. We have identified the following concrete reasons why these combinations make TEP imaging suitable in studying exocytosis and endocytosis.

Two-photon excitation is inherently resistant to light scattering in tissue preparations [16], and we could study fine structural alteration in tissue without much sacrificing the spatial resolution of the microscope. In TEP imaging, we immerse tissues in a solution containing polar tracer, and one can worry that signal-tonoise ratio is compromised with the presence of large amount of tracers. This is not the case, because intercellular space of tissues is normally as narrow as 20 $40 \mathrm{~nm}$, which is less than the diameter of most secretory vesicles (Fig. 1d). In addition, the intercellular space within tissue is very clean, unlike the space between a cell and glass coverslip. Thus, the intercellular space provides one of the best experimental systems to study exocytosis, not to mention the fact that it is the physiological site for secretion.

Two-photon excitation does not excite tracer mole- cules outside of the focal plane, and allows the use of high concentrations of polar tracers that are necessary to detect fine alterations in cellular structures. With one-photon excitation, heat and phototoxic effects are generated in the entire tissues, and damage the preparations. The photobleaching of tracers in the focal plane is effectively compensated for by diffusion of tracer molecules from outside of the focal plane. In conventional illumination, fluorescent probes are continuously photobleached in the entire depth of preparations during image acquisition, and bleaching cannot be overcome by diffusion. Therefore, absolute values of fluorescence intensity cannot be utilized for quantification of exocytosis in conventional imaging.

In one photon excitation, actual excitation beam might be reduced by high concentrations of polar tracers present between the objective lens and the focal plane. The inner filter effect was particularly problematic when we observe structures deep in the tissue, and when we perform multicolor imaging. In two-photon excitation, there is little absorption of excitation beam except for the focal plane, and there is no inner filter effect for the excitation beam.

We can ideally control the state and concentration of tracers in postfusion labeling, since polar tracers are directly applied to extracellular spaces. In contrast, it is difficult to control the state of the probe in prefusion labeling (Fig. 1c), since their properties are likely modified after they are incorporated into vesicles. The prefusion labeling is therefore a not good way to characterize exocytosis per se, although it is necessary to track the history of vesicle before exocytosis. Postfusion labeling can stain exocytic vesicles with simple diffusion without selection bias. SRB can fill in vesicles fairly rapidly even in the case of small vesicles where narrow fusion pore open only transiently [12]. Thus, TEP imaging reveals all exocytic events in the visual field [13], and characterizes abnormality of secretion in diseased or mutant animals without selection bias.

Finally, two-photon excitation can simultaneously excite multiple tracers with single laser source due to broader two-photon excitation spectra by the blue shift in the two-photon excitation spectra as relative to the simple prediction. Usage of the same laser beam eliminates the offsets caused by chromatic aberration and by alignment of two laser beams. In addition, we do not use pin-hole in two-photon excitation, and detect most of the fluorescence with photomultiplier, and 
therefore the collection of fluorescent light is little affected by wavelength. Thus, two-photon imaging enables completely simultaneous multicolor imaging without spatial offset. With the simultaneous multicolor imaging, we can measure precise time difference of the fluorescent events, and perform ratiometric imaging to obtain internal states of molecule and cell.

Exocytosis detected by TEP imaging is so definitive that the onset of fusion of individual vesicles can be defined, the diameter of vesicle measured, and the fates of vesicles after exocytosis followed. TEP imaging can thus be used as standalone technique to study exocytosis and endocytosis. TEP imaging can be successfully combined with capacitance measurement [12] and electron microscopic investigation [11, 12] when necessary.

\section{Nanometer measurement of diameter of exocytic and endocytic vesicles}

Identification of vesicle types is fundamental in study of exocytosis, since multiple types of exocytic and endocytic vesicles are functioning even in single cells. The measurement of vesicle diameter is not a trivial problem, because the diameters are close to or less than the spatial resolution of optical microscopes. The capacitance measurements, one of the most popular methods for quantifying exocytosis, also cannot discriminate the signal of the different types of granules. TEP imaging allows us to measure diameters of vesicles, by comparing fluorescence intensity of individual exocytic events with that of extracellular space (Fig. 3). TEP imaging can also characterize the fusion pore, since exocytosis can be simultaneously monitored by multiple tracers with different colors. These approaches have been collectively termed as TEP imaging-based quantitation (TEPIQ). Note that, if one-photon confocal imaging is used for TEPIQ like analysis, heat generated by one-photon absorption throughout the tissue will damage the tissues when we use sufficient laser power to detect the small vesicles. TEPIQ analyses of vesicle diameters have three variants as reviewed [17]. Briefly, the three methods are 1) to predict the volume of exocytic vesicles $(\Delta V)$ from the stepwise increase in fluorescence of polar tracer such as sulforhodamine $B, 2$ ) to estimate the surface area $(\Delta S)$ of vesicles from the stepwise increase in membrane-staining fluorescence $\left(F_{\mathrm{S}}\right)$ such as

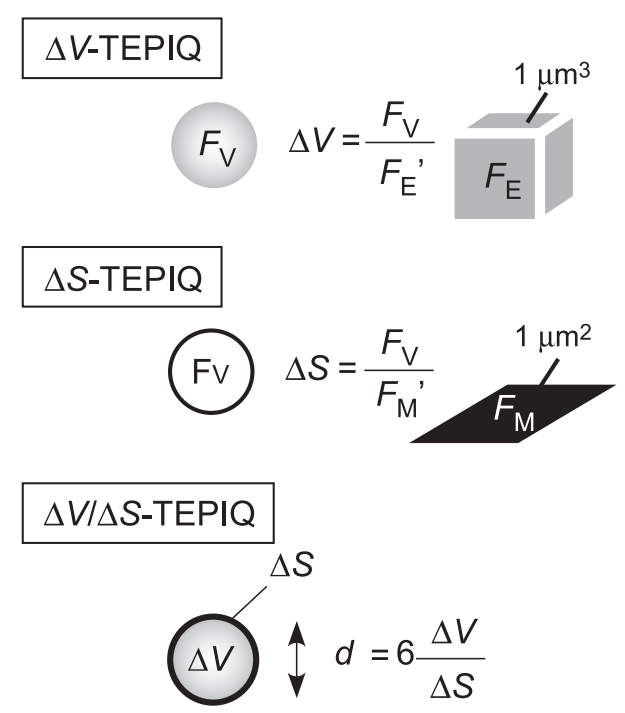

Fig. 3. Three variations of TEPIQ analysis for diameters of exocytic vesicles [10].

FM1-43, and 3) to estimate the diameter from the ratio between volume and surface area, because the diameter $(d)$ of vesicles was estimated as estimated as $d=$ $6 \Delta V / \Delta S$ given that

$$
6 \times \frac{4}{3} \pi r^{3} / 4 \pi r^{2}=2 r=d[10] .
$$

According to the optical resolution of microscopy, conventional full-width at half-maximal (FWHM) diameters of fluorescent vesicles cannot be used to estimate diameters of vesicles less than $0.4 \mu \mathrm{m}$, where they are nearly insensitive to the actual diameter. Using TEPIQ analysis of $\Delta V$, however, diameters of large dense-core vesicles of PC12 cells, $\beta$-cells and adrenal chromaffin cells have been estimated as $220 \mathrm{~nm}$, $350 \mathrm{~nm}$ and $500 \mathrm{~nm}$, respectively, which are consistent with those reported by electron microscope $[10,11$, 14]. TEPIQ analysis of $\Delta V / \Delta S$ have also estimated the diameters of small vesicles, kiss-and-run endocytic vesicles and clathrin-mediated endocytic vesicles of PC12 cells, as $55 \mathrm{~nm}, 55 \mathrm{~nm}$ and $90 \mathrm{~nm}$ [12]. These diameters were consistent with those estimated with electron microscopy using photoconversion of FM143 [12].

\section{Fusion pore dynamics at nanometer resolution}

The fusion pore is the initial aqueous pore formed 
by fusion of two membranes, vesicle membrane and plasma membrane. Formation of the fusion pore is key to understand fusion reaction. Moreover, the fates of the fusion pore determine those of vesicles. The fusion pore can dilate infinitely to induce full fusion exocytosis, as we will describe below [7]. It can stably stay for a long time, and induce sequential exocytosis $[6,9,11,14]$, or can be closed again for kiss-and-run or stay endocytosis [12].

Time-resolved membrane capacitance measurement estimated the initial diameter of the pore (Fig. 1a), however, it cannot estimate the diameters of pore larger than $2 \mathrm{~nm}$ and $6 \mathrm{~nm}$ for small and large vesicles [18], respectively, and may underestimate the pore size. Amperometric measurement cannot by itself estimate the diameter of the fusion pore (Fig. 1b). It was therefore useful to develop imaging approach to measure fusion pore diameter. We thus used fluorescent polar tracers as nanometer sized probes (Fig. 4a). If the pore is larger than the effective diameter of probe, the vesicle will be stained.

We therefore placed two polar tracers, for example, SRB $(0.3-0.7 \mathrm{mM})$ and dextrans conjugated with fluorescein $(0.5-2 \mathrm{mM})$ with a variety of molecular weights. Based on the molecular structures and light scattering, we estimate the hydrodynamic diameters of SRB and $10 \mathrm{k}-\mathrm{Da} \mathrm{FD}$ as $1.4 \mathrm{~nm}$ and $6 \mathrm{~nm}$, respectively (Fig. $4 a)$. We found that large dense-core vesicles of adrenal chromaffin cells and PC12 cells were nearly simultaneously stained with the two compounds within a time lag less than $50 \mathrm{~ms}$, consistent with the fact that the lifetime of the fusion pore of $10-50 \mathrm{~ms}$ in these cells studied with capacitance measurement and amperometry [19]. We also found that the fusion pore was reversibly closed without further opening to larger than $6 \mathrm{~nm}[7,11,14]$, which indicate that the pores are semistable and reversibly closed again. These are consist with the original notion of the fusion pore, and proved that the fusion pore plays important role also in intact tissues.

In $\beta$-cells, however, there were significant time lag between SRB and $10 \mathrm{k}-\mathrm{Da}$ FD signals of 1-2 $\mathrm{s}$ with the mean value of $1.8 \mathrm{~s}$ (Fig. 4b, c) [7], suggesting that dilation of the fusion pore is exceptionally slow in insulin vesicles of $\beta$-cells. Consistent with this idea, the time course of staining of vesicles with polar tracers was slower than other cells (Fig. 4b). The following observations suggest that this was due to crystallization of insulin in the granules that prevented the dila-

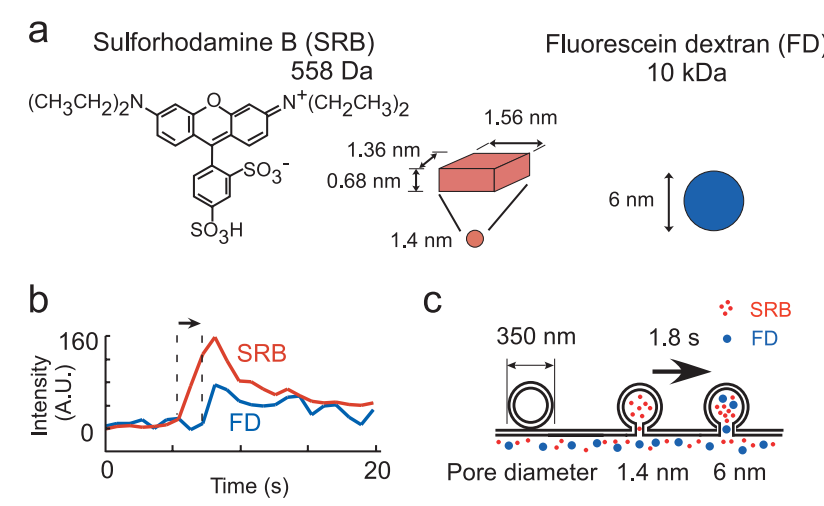

Fig. 4. TEPIQ analysis of fusion-pore expansion in insulin vesicle. a) Molecular dimensions of SRB and 10-kDa dextran conjugated with fluorescein (FD). The length of the second longest side of SRB $(1.4 \mathrm{~nm})$ is used for the effective diameter. b) Time courses of single exocytic event of insulin vesicle stained with both SRB (red) and FD (blue). c) Schematic drawing of the expansion of the fusion pore.

tion of granule content and the fusion pore. First, the pore dilation was further retarded by adding zinc $(3 \mathrm{mM})$ to the extracellular solution [7], which is known to stabilize insulin crystal by binding to insulin [20]. Second, the pore dilation was significantly faster in the islets of guinea pig, where crystallization of insulin is known to be weak [20]. Finally, flattening of vesicles was found to start after when the fusion pores were dilated to more than $12 \mathrm{~nm}$, which was confirmed by FD with molecular weight of $70 \mathrm{kDa}$. This finding may suggest that the flattening of vesicle occur when fusion pore allow permeation of insulin hexamer with the molecular weight of $36 \mathrm{kDa}$.

\section{Analysis of fusion-pore composition}

The slow dilation of the fusion pore of insulin vesicles allowed us to examine its molecular composition. It is worthwhile seeing the time course of staining of insulin vesicles with lipidic dye FM1-43, since the pore size remained small for a few seconds. If the fusion pore is proteinaceous, aqueous pore is the only pathway for FM1-43 staining (Fig. 5a, red). Staining of vesicles with FM1-43 should be similar or slower than SRB, since FM1-43 accumulates in the plasma membrane. In contrast, if the fusion pore is lipidic, FM1-43 can stain insulin vesicles via lateral diffusion from the already stained plasma membrane (Fig. 5a, 


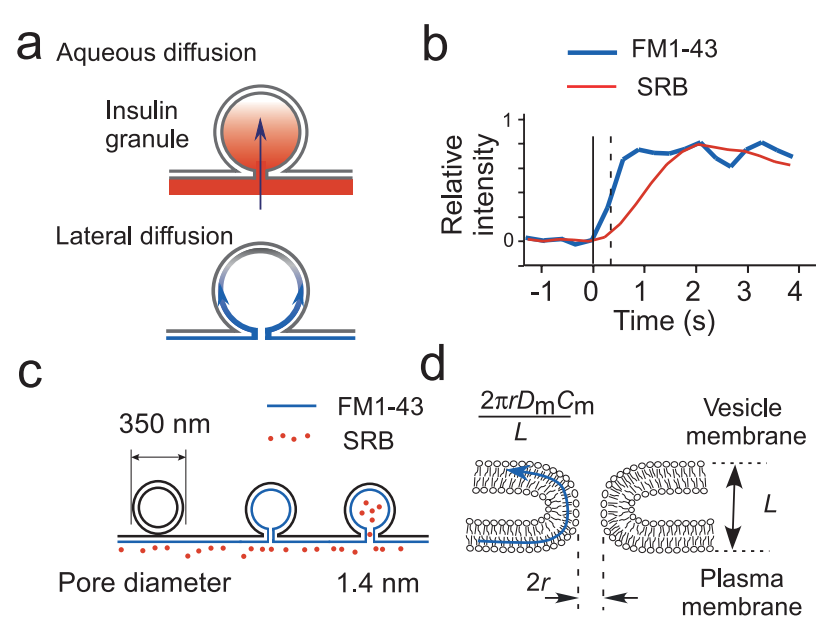

Fig. 5. TEPIQ analysis of fusion-pore composition of insulin vesicle. a) Two possible routes of staining of a vesicle with FM1-43. b) Time courses of single exocytic event of insulin vesicle stained with both SRB (red) and FM143 (blue). c) Schematic drawing of vesicle staining with FM1-43 and SRB. d) Flux of FM1-43 molecule along the lateral wall of the fusion pore [7].

blue). Since the vesicle is as small as $350 \mathrm{~nm}$, the lateral diffusion should rapidly be induced dependent on the geometry of the fusion pore.

We found that FM1-43 stained the vesicle with an earlier onset and faster time course than SRB (Fig. 5b, c) $[7,10]$. More precisely, FM1-43 stained the vesicle more than $64 \%$ when SRB signal just started to appear. We estimated the diffusion constant of FM1-43 molecule along the fusion pore (Fig. $5 \mathrm{~d}$ ) as $3.3 \mu \mathrm{m}^{2} / \mathrm{s}$ [7]. This value is within the range for pure lipid bilayer, while is larger than that in the plasma membrane $\left(<1 \mu \mathrm{m}^{2} / \mathrm{s}\right)$, where lateral diffusion of membrane lipid is prevented by actin-based membrane skeleton meshwork with the "picket" mechanism [21]. This indicates that the fusion pore is already lipidic when the pore size is about $1.4 \mathrm{~nm}$. This precludes the purely proteinacious fusion pore model, and the proteins, though existent, should not much disturbed the flow of lipid molecule, like FM1-43. The faster staining of vesicle by FM1-43 than SRB has been seen only in insulin granules, but not in the large dense-core vesicles of PC12 cells [11] and adrenal chromaffin cells [14]. This is because the pore expands rapidly in these cells, and vesicles are also rapidly stained with SRB in these cells.

\section{Full-fusion exocytosis of insulin vesicles}

We confirmed that TEP-imaging visualizes most insulin exocytosis in a focal plane of the islet, as predicted amount of insulin secretion from the number of glucose-induced exocytic events can account for the amount of insulin release by RIA [13]. Based on TEPIQ analysis, we are sure that the events reflect large dense-core vesicles of $\beta$-cells. We can therefore safely characterize the typical properties of insulin exocytosis. Somewhat unexpectedly, exocytosis was found in all over the plasma membrane of $\beta$-cells, and there is only a slight inclination for exocytosis to occur preferentially towards the blood vessels [7]. This is consistent with the fact that no tight-junction structure is seen in islets and endocrine cells in general, and indicates that extracellular space is the major pathway for secretion of hormone.

We found that most of them underwent full flattening with the plasma membrane in $92 \%$ of glucoseinduced events [7], though stable omega structures were formed when larger increase in cytosolic calcium concentration was given. The full fusion exocytosis is the simplest form of exocytosis, and has been assumed for a long time, but TEP-imaging has, for the first time, definitively demonstrated its actual use in mammalian secretory cells. The full fusion occurred with two time constants, $1.5 \mathrm{~s}$ and $15 \mathrm{~s}$ in $\beta$-cells, somewhat slower than chromaffin cells, where its life was about $0.25 \mathrm{~s}$ [14], possibly owing to the time for dissolution of insulin crystal to hexamer. In fact, it has become evident that $\beta$-cells are rather exceptional in showing predominantly full fusion exocytosis, and other two forms of exocytosis, sequential (2\%) and kiss-and-run exocytosis $(6 \%)$ were relatively infrequent. We think that kiss-and-run exocytosis is suppressed in $\beta$-cells, since such transient opening of fusion pore does little to insulin secretion. Protein kinase A further reduced kiss-and-run exocytosis in $\beta$-cells [13]. We think that sequential exocytosis (Fig. 6a) is suppressed in $\beta$-cells, for insulin exocytosis to be more sensitive to energy states of the cells [8]. One might imagine that sequential exocytosis is infrequent in $\beta$-cells, simply because most vesicles undergo full fusion, and give no chance for secondary exocytosis. This is, however, not the case, since stimulation with caged-Ca ${ }^{2+}$ compound greatly prolonged the lifetime of $\Omega$-shaped profiles, while it did not change the occurrence of sequential exocytosis [8]. 
TEP imaging also fit nicely with molecular biology, as we can directly study the tissues of mutant animals. We have succeeded in reproducing results of RIA of insulin secretion in three mutants mice, ashen mice (lacking Rab27a) [22], collectrin transgenic mice [23] and $\beta$-cell-specific HNF- $4 \alpha$ knockout mice [24]. Although abnormality in spatial organizations of insulin exocytosis was not found in these animals where single genes were mutated, it does not rule out the possible impairment of spatial organizations of exocytosis in real diabetic islets where wide variety of genes and metabolisms are affected.

\section{Involvement of protein kinase A in insulin exocytosis}

Insulin secretion is known to occur in two phases in the islet after glucose stimulation. The first phase (2$7 \mathrm{~min}$ ) of insulin secretion directly triggers glycogen synthesis in the liver, and is considered to play an important role in the control of the blood glucose level, and is particularly impaired in type 2 diabetes mellitus. There must be many underlying mechanisms for the two phases of insulin secretion. Using TEP imaging we have revealed that protein kinase A (PKA) is specifically involved in the initial period of the first phase of insulin exocytosis [13]. We found that a series of hydrophilic PKA inhibitors (PKI, Rp-cAMPS) potently and particularly blocked the initial period of the first phase of insulin exocytosis from the intact islet. Previously, it has been reported that PKA antagonists have no effect or small effect on secretion based on RIA $[25,26]$. It is therefore now suggested that the negative effect is due to the lack of time resolution, which results in an underestimation of the contribution of the initial period of insulin exocytosis. It is also due to the lack of awareness that membrane permeable inhibitors often do not penetrate into tissues when applied by superfusion [15], because we have demonstrated certain PKA inhibitors (H89 and KT5720) was effective only in small cluster preparations, not in the whole islets [13]. We have noticed the problem as the result of direct observation of tissues and exocytosis. We also estimated the $\mathrm{Ca}^{2+}$ signaling together with TEP imaging, and found that these inhibitors did not affect the increases in cytosolic $\mathrm{Ca}^{2+}$ induced by glucose [13]. It was therefore suggested that PKA should have direct effects on exocytosis.
TEP imaging can also be used with one of the most powerful tools of modern biophysics, caged compounds, where signaling mechanisms can be switched on in a millisecond time resolution by a flash light. We examined the exocytosis induced by flash photolysis of caged $\mathrm{Ca}^{2+}$ compound loaded into the islet in the form of AM-ester $[8,13]$. Rapid and large increases in $\mathrm{Ca}^{2+}$ induced exocytosis occurred within $1 \mathrm{~s}$, and decayed with two time constants of approximately $1 \mathrm{~s}$ and $10 \mathrm{~s}$ as reported by amperometry [27, 28]. We examined the effects of glucose on insulin exocytosis by applying uncaging before high glucose by itself induced increases in cytosolic $\mathrm{Ca}^{2+}$ concentrations and exocytosis, and found that glucose potentiated the uncaging induced exocytosis [13]. Thus, glucose induced facilitation of insulin exocytosis without increases in concentrations of cytosolic $\mathrm{Ca}^{2+}$, consistent with the notion of the K-ATP channel-independent action of glucose [29-31]. The glucose effect was completely eliminated by PKA inhibitors [13]. Interestingly, forskolin, which increases cytosolic concentrations of cAMP, potentiated exocytosis only at a high glucose concentration, but not at low glucose, indicating that a factor other than cAMP is also required for PKA activity. One possible candidate is cytosolic ATP, which is known to be increased by glucose, and which is known to potentiate insulin exocytosis in a manner dependent on PKA [28]. Thus, these experiments indicate that PKA is involved in the glucose sensing mechanism.

Exactly how PKA regulates exocytosis remains to be elucidated. We have revealed that PKA reduces kiss-and-run exocytosis [13] and affects the fates of the fusion pore, suggesting that targets of PKA may involve the final fusion reaction.

\section{Mode of exocytosis}

Compound exocytosis is classified into two types. One type is sequential exocytosis, which we found in exocrine pancreas by TEP imaging [6]. The $\Omega$-shaped profiles in pancreatic acinar cells were stable for several minutes, and deep vesicles selectively fused with the granule membrane which was already fused with the plasma membrane (Fig. 6a, c). The chain reaction proceeded deep into the cytosol in an apparently random manner, as a thunder, with the speeds of fusion was not slower in the deep cytosolic layer as relative to 
those in the surface. We looked for relevant literatures, and found that we must have revealed dynamic sequence of events whose static analysis was reported in 1965 with electron microscope and named "sequential exocytosis" [32]. In endocrine tissues, sequential exocytosis was frequently observed in adrenal medulla (63\% of the total exocytic events) [14], but severely inhibited in pancreatic islet (2\%) [8].

There is another type of compound exocytosis, called multivesicular (or multigranular) exocytosis, where vesicles fused each others in the cytosol before the vesicles to fuse with the plasma membrane (Fig. $6 \mathrm{~b})$. This type of compound exocytosis has been known to be utilized in hematopoietic cells (Fig. 6d). Secretion can be explosive in multivesicular exocytosis (Fig. 6d), while secretion is under full control of agonist in sequential exocytosis (Fig. 6c). We think multivesicular compound exocytosis rarely happens in pancreatic islet, acinar cells, and adrenal medulla, since we have seldom found sudden appearance of compound vesicles (Fig. 6b).

Note that the term "compound exocytosis" was used specifically for sequential exocytosis in an early study of mast cell [33], while for multivesicular exocytosis in studies of eosinophils [34, 35]. More recently, the term "compound exocytosis" has been used to include both sequential and multivesicular exocytosis $[8,36]$. Compound exocytosis was reported in studies of anterior pituitary cell [37, 38], alveolar type II cell [39], and mast cell, without distinguishing sequential and multivesicular exocytosis. Both types of exocytosis must exist in hematopoietic cells $[34,35]$ and might be present in parotid acinar cells.

\section{Lateral diffusion of SNARE proteins during exocytosis}

The fact that compound exocytosis was strictly sequential in pancreatic acinar cells [6], islet $\beta$-cells [8], adrenal chromaffin cells [14] and PC12 cells [11] even when spatially homogenous increase in cytosolic $\mathrm{Ca}^{2+}$ concentrations suggests that some factors necessary for fusion diffuse from the plasma membrane into the vesicle membrane (Fig. 6a). For the strict sequential exocytosis to happen, vesicles in the cytosol needs to recognize the vesicles which are continuous with the plasma membrane for fusion, and the mechanism how this recognition can be taken place has been a well a Sequential exocytosis

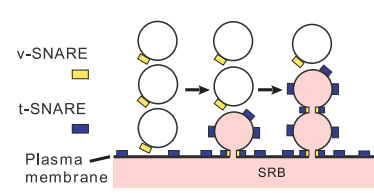

b Multivesicular exocytosis

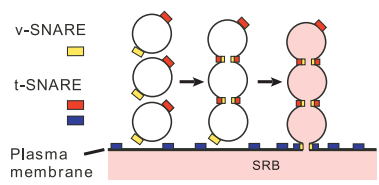

C

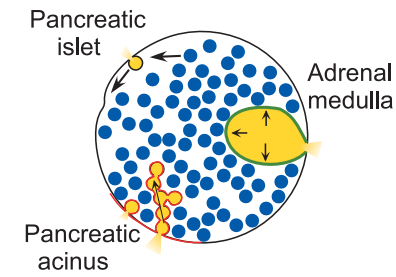

d Hematopoietic cells

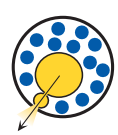

Fig. 6. Two forms of compound exocytosis. a) Sequential exocytosis is supported by lateral diffusion of t-SNARE from the plasma membrane [6]. b) Multivesicular exocytosis may utilize two distinct t-SNAREs. c) Fullfusion exocytosis, sequential exocytosis and vacuolar sequential exocytosis detected in the pancreatic islet, acinus and adrenal medulla, respectively. Red outlines in pancreatic acinus indicating actin coating. d) Multivesicular exocytosis of eosinophil and basophil [17].

defined question in the field of exocytosis. We have therefore proposed that target SNARE proteins ( $t$ SNARE) may diffuse from the plasma membrane to granule membrane, and trigger sequential fusion events (Fig. 6a) [6]. Such possibility was tested in islet $\beta$-cells [8] and in adrenal chromaffin cells [14].

Sequential exocytosis was prevalent and rapid in adrenal chromaffin cells, where lateral diffusion of SNAP- 25 was detected in $43 \%$ of exocytic events, and occurred with only with a short latency of $1.15 \mathrm{~s}$ from primary exocytosis. In contrast in $\beta$-cells, lateral diffusion of SNAP- 25 was detected only in $6 \%$ of exocytic events, and occurred with a long latency of $6.5 \mathrm{~s}$, where sequential exocytosis was slow and infrequent (2\%). Importantly, lateral diffusion of SNAP-25 was still detected in $54 \%$ of vesicles undergoing sequential exocytosis in $\beta$-cells, but only $5 \%$ in solitary exocytic events. Furthermore, when islets were treated with cyclodextrin to remove lipid raft, which might trap SNAP-25, sequential exocytosis was hastened and increased to $8.9 \%$, and lateral diffusion of SNAP-25 was detected in $15 \%$ of events [8]. Thus, lateral diffusion of SNAP-25 showed parallelism with the sequential progression of exocytosis in chromaffin cells and $\beta$-cells. Sequential exocytosis also involves other SNAREs, as redistribution of syntaxin-2 has been demonstrated by immunohistochemistry in pancreatic acinar cells. 
Assembling of SNARE complex has been often considered to precede the final triggers to account for rapid exocytosis, because SNARE assembling was reported to take more than $60 \mathrm{~s}$ in vitro [40]. Our data in chromaffin cells indicate that the complex formation could occur at least within in a second. Protein folding and assembling are known to be speeded up with molecular chaperons, and SNARE assembling may occur more rapidly in the cytosol than in vitro. It must be important to identify which molecules act as a molecular chaperon for SNARE complex formation.

\section{Summary}

We hope that the examples described in this review vitally explain the power of TEP imaging in investigating exocytosis and endocytosis of endocrine tissues and nervous systems. TEP imaging is the only method that can investigate intact secretory tissues, and can address almost every important issue of exocytosis, including vesicle diameters, fusion-readiness, fusionpore properties, life of $\Omega$-shaped profiles, swelling of vesicles, compound exocytosis, endocytosis, spatial organizations of exocytosis, proteins involved in exocytosis, such as SNARE proteins and actin cytoskeletons. Such imaging technique is suited to study mutant animals and even human specimens, because we can study tissue preparations. It will be also possible to perform simultaneous prefusion and postfusion labeling to track the entire life of vesicles before and after exocytosis in future, and we hope this method to be used for wide varieties of medial and pharmaceutical applications.

\section{Acknowledgements}

We thank Tomomi Nemoto, Takuya Kishimoto, Ting-Ting Liu, Hiroyasu Hatakeyama and the members of our laboratory for the experiments and valuable discussions.

\section{References}

1. Augustine GJ, Burns ME, DeBello WM, Pettit DL, Schweizer FE (1996) Exocytosis: Proteins and perturbations. Annu Rev Pharmac Toxicol 36: 659-701.

2. Kasai H (1999) Comparative biology of exocytosis: Implications of kinetic diversity for secretory function. Trends Neurosci 22: 88-93.

3. Augustine GJ (2001) How does calcium trigger neurotransmitter release? Curr Opin Neurobiol 11: 320-326.

4. Seino S, Shibasaki T (2005) PKA-dependent and PKAindependent pathways for cAMP-regulated exocytosis. Physiol Rev 85: 1303-1342.

5. Jahn R, Lang T, Südhof TC (2003) Membrane fusion. Cell 112: 519-533.

6. Nemoto T, Kimura R, Ito K, Tachikawa A, Miyashita Y, Iino M, Kasai H (2001) Sequential-replenishment mechanism of exocytosis in pancreatic acini. Nat Cell Biol 3: 253-258.

7. Takahashi N, Kishimoto T, Nemoto T, Kadowaki T, Kasai H (2002) Fusion pore dynamics and insulin granule exocytosis in the pancreatic islet. Science 297: 1349-1352.

8. Takahashi N, Hatakeyama H, Okado H, Miwa A, Kishimoto T, Kojima T, Abe T, Kasai H (2004) Sequential exocytosis of insulin granules is associated with redistribution of SNAP25. J Cell Biol 165: 255262.

9. Oshima A, Kojima T, Dejima K, Hisa I, Kasai H,
Nemoto T (2005) Two-photon microscopic analysis of acetylcholine-induced mucus secretion in guinea pig nasal glands. Cell Calcium 37: 349-357.

10. Kasai H, Hatakeyama H, Kishimoto T, Liu TT, Nemoto T, Takahashi N (2005) A new quantitative (two-photon extracellular polar-tracer imaging-based quantification (TEPIQ)) analysis for diameters of exocytic vesicles and its application to mouse pancreatic islets. J Physiol 568: 891-903.

11. Kishimoto $\mathrm{T}$, Liu TT, Hatakeyama $H$, Nemoto $\mathrm{T}$, Takahashi N, Kasai H (2005) Sequential compound exocytosis of large dense-core vesicles in PC12 cells studied with TEPIQ (two-photon extracellular polartracer imaging-based quantification) analysis. $J$ Physiol 568: 905-915.

12. Liu TT, Kishimoto $T$, Hatakeyama $H$, Nemoto $T$, Takahashi N, Kasai H (2005) Exocytosis and endocytosis of small vesicles in PC12 cells studied with TEPIQ (two-photon extracellular polar-tracer imagingbased quantification) analysis. J Physiol 568: 917-929.

13. Hatakeyama H, Kishimoto $\mathrm{T}$, Nemoto $\mathrm{T}$, Kasai $\mathrm{H}$, Takahashi N (2006) Rapid glucose sensing by protein kinase A for insulin exocytosis in mouse pancreatic islets. J Physiol 570: 271-282.

14. Kishimoto T, Kimura R, Liu TT, Nemoto T, Takahashi N, Kasai H (2006) Vacuolar sequential exocytosis of large dense-core vesicles in adrenal medulla. EMBO J 
25: 673-682.

15. Takahashi N, Nemoto $\mathrm{T}$, Kimura R, Tachikawa A, Miwa A, Okado H, Miyashita Y, Iino M, Kadowaki T, Kasai H (2002) Two-photon excitation imaging of pancreatic islets with various fluorescent probes. Diabetes 51 (Suppl 1): S25-S28.

16. Dong CY, Koenig K, So P (2003) Characterizing point spread functions of two-photon fluorescence microscopy in turbid medium. J Biomed Opt 8: 450-459.

17. Kasai H, Kishimoto T, Nemoto T, Hatakeyama H, Liu TT, Takahashi N (2006) Two-photon excitation imaging of exocytosis and endocytosis and determination of their spatial organization. Adv Drug Deliver Rev 58: 850-877.

18. Klyachko VA, Jackson MB (2002) Capacitance steps and fusion pores of small and large-dense-core vesicles in nerve terminals. Nature 418: 89-92.

19. Breckenridge LJ, Almers W (1987) Currents through the fusion pore that forms during exocytosis of a secretory vesicle. Nature 328: 814-817.

20. Dodson G, Steiner D (1998) The role of assembly in insulin's biosynthesis. Curr Opin Struct Biol 8: 189-194.

21. Fujiwara T, Ritchie K, Murakoshi H, Jacobson K, Kusumi A (2002) Phospholipids undergo hop diffusion in compartmentalized cell membrane. J Cell Biol 157: 1071-1082.

22. Kasai K, Ohara-Imaizumi M, Takahashi N, Mizutani S, Zhao S, Kikuta T, Kasai H, Nagamatsu S, Gomi H, Izumi $\mathrm{T}$ (2005) Rab27a mediates the tight docking of insulin granules onto the plasma membrane during glucose stimulation. J Clin Invest 115: 388-396.

23. Fukui K, Yang Q, Cao Y, Takahashi N, Hatakeyama H, Wang H, Wada J, Zhang Y, Marselli L, Nammo T, Yoneda K, Onishi M, Higashiyama S, Matsuzawa Y, Gonzalez FJ, Weir GC, Kasai H, Shimomura I, Miyagawa J, Wollheim CB, Yamagata K (2005) The HNF-1 target collectrin controls insulin exocytosis by SNARE complex formation. Cell Metab 2: 373-384.

24. Miura A, Yamagata K, Kakei M, Hatakeyama H, Takahashi N, Fukui K, Nammo T, Yoneda K, Inoue Y, Sladek FM, Magnuson MA, Kasai H, Miyagawa J, Gonzalez FJ, Shimomura I (2006) Hepatocyte nuclear factor-4alpha is essential for glucose-stimulated insulin secretion by pancreatic beta-cells. J Biol Chem 281: 5246-5257.

25. Persaud SJ, Jones PM, Howell SL (1990) Glucosestimulated insulin secretion is not dependent on activation of protein kinase A. Biochem Biophys Res Com 173: 833-839.

26. Harris TE, Persaud SJ, Jones PM (1997) Pseudosubstrate inhibition of cyclic AMP-dependent protein ki- nase in intact pancreatic islets: effects on cyclic AMPdependent and glucose-dependent insulin secretion. Biochem Biophys Res Com 232: 648-651.

27. Takahashi N, Kadowaki T, Yazaki Y, Miyashita Y, Kasai K (1997) Multiple exocytotic pathways in pancreatic $\beta$ cells. $J$ Cell Biol 138: 55-64.

28. Takahashi N, Kadowaki T, Yazaki Y, Ellis-Davies GCR, Miyashita Y, Kasai H (1999) Post-priming actions of ATP in the $\mathrm{Ca}^{2+}$ dependent exocytosis in pancreatic $\beta$-cells. Proc Natl Acad Sci USA 96: 760 765.

29. Gembal M, Gilon P, Henquin J (1992) Evidence that glucose can control insulin release independently from its action on ATP-sensitive $\mathrm{K}^{+}$channels in mouse B cells. J Clin Invest 89: 1288-1295.

30. Aizawa T, Sato Y, Ishihara F, Taguchi N, Komatsu M, Suzuki N, Hashizume K, Yamada T (1994) ATPsensitive $\mathrm{K}^{+}$channel-independent glucose action in rat pancreatic $\beta$-cell. Am J Physiol 266: C622-C627.

31. Henquin JC (2000) Triggering and amplifying pathways of regulation of insulin secretion by glucose. Diabetes 49: 1751-1760.

32. Ichikawa A (1965) Fine structural changes in response to hormonal stimulation of the perfused canine pancreas. J Cell Biol 24: 369-385.

33. Alvarez de Toledo G, Fernandez JM (1990) Compound versus multigranular exocytosis in peritoneal mast cells. J Gen Physiol 95: 397-409.

34. Scepek S, Lindau M (1993) Focal exocytosis by eosinophils - compound exocytosis and cumulative fusion. EMBO J 12: 1811-1817.

35. Hafez I, Stolpe A, Lindau M (2003) Compound exocytosis and cumulative fusion in eosinophils. $\mathrm{J} \mathrm{Biol} \mathrm{Chem}$ 278: 44921-44928.

36. Pickett JA, Edwardson JM (2006) Compound exocytosis: mechanisms and functional significance. Traffic 7 : 109-116.

37. Fujita H, Kurihara H, Miyagawa J (1983) Ultrastructural aspect of the effect of calcium ionophore A23187 on incubated anterior pituitary cells of rats. Cell Tissue Res 229: 129-136.

38. Cochilla AJ, Angelson JK, Betz WJ (2000) Differential regulation of granule-to-granule and granule-to-plasma membrane fusion during secretion from rat pituitary lactotrhophs. J Cell Biol 150: 839-848.

39. Mair N, Haller T, Dietl P (1999) Exocytosis in alveolar type II cells revealed by cell capacitance and fluorescence measurements. Am J Physiol 276 : L376-382.

40. Fasshauer D, Antonin W, Subramaniam V, Jahn R (2002) SNARE assembly and disassembly exhibit a pronounced hysteresis. Nat Struct Biol 9: 144-151. 